\title{
RECEIVED
}

\section{APR 141997}

\section{OSTI}

\author{
TESTS OF QCD AT HERA: \\ Determination of the Gluon Density
}

\author{
JOSE REPOND \\ Argonne National Laboratory
}

\begin{abstract}
An overview is given of the various methods available to the colliding beam experiments at HERA to determine the gluon density of the proton. The article includes a description of fits to the structure function $F_{2}$, of studies of dijet and open charm production in deep inelastic scattering, of elastic and inelastic $\psi$ photoproduction, and of inclusive diffractive scattering.
\end{abstract}

\section{Introduction}

Overviews of recent results concerning many physics topics studied by the collider experiments $\mathrm{HI}$ and ZEUS at HERA have been given elsewhere ${ }^{1}$. In this talk we concentrate on the central topic of determining the gluon density, $x g(x)$, in the proton.

Since the gluon carries no electric charge, all methods of determining $x g(x)$ in lepton-proton scattering are somewhat indirect. As a consequence, the methods are subject to relatively large systematic uncertainties. Therefore, it is essential to determine $x g(x)$ by as many different analysis techniques as possible. Since the techniques have very different systematic uncertainties, the comparison of their results will give significantly more confidence in the determination of $x g(x)$ and can be viewed as a stringent test of the validity of QCD.

The different methods of extracting $x g(x)$ at HERA can be classified into three categories:

- In gluon exchange, where $n$ means any number of gluons: this category contains the next-to-leading order (NLO) QCD fits to the proton structure function $F_{2}$. Currently this is the most precise method extending over a large kinematic region. In the same category falls the measurement of the longitudinal structure function, $F_{L}$, which is directly related to $x g(x)$. Unfortunately, the measurement of $F_{L}$ is very difficult, requiring precise measurements of $F_{2}$ at different beam energy settings. Such measurements are not yet available.

- 1 gluon exchange: this category contains the photon-gluon fusion (PGF) process, in which the photon interacts with a gluon emitted from 


\section{DISCLAIMER}

This report was prepared as an account of work sponsored by an agency of the United States Government. Neither the United States Government nor any agency thereof, nor any of their employees, make any warranty, express or implied, or assumes any legal liability or responsibility for the accuracy, completeness, or usefulness of any information, apparatus, product, or process disclosed, or represents that its use would not infringe privately owned rights. Reference herein to any specific commercial product, process, or service by trade name, trademark, manufacturer, or otherwise does not necessarily constitute or imply its endorsement, recommendation, or favoring by the United States Government or any agency thereof. The views and opinions of authors expressed herein do not necessarily state or reflect those of the United States Government or any agency thereof. 


\section{DISCLAIMER}

Portions of this document may be illegible in electronic image products. Images are produced from the best available original document. 
the proton via a quark anti-quark loop. The rate of this process is proportional to $x g(x)$. The PGF process can be tagged by requiring dijets, open charm or inelastic $\psi$ 's in the hadronic final state.

- 2 gluon exchange: two processes can be included in this category: exclusive vector meson production and inclusive diffractive scattering. If the 2 gluon exchange mechanism is indeed responsible for these processes, currently a matter of heated debates ${ }^{2}$, their cross sections will be proportional to $[x g(x)]^{2}$.

In the following section we shall briefly describe the different methods and then compare their results in the concluding chapter.

\section{Experimental Results}

\subsection{The collider experiments at HERA}

Since its commissioning in 1992, HERA has delivered a total integral luminosity of about $22 \mathrm{pb}^{-1}$. The results presented in this talk were obtained by the two colliding beam experiments, H1 and ZEUS, from data collected during the 1994 running period from collisions of $27.5 \mathrm{GeV} e^{+}$with $820 \mathrm{GeV} p$. These data sets correspond each to an integrated luminosity of approximately $3 \mathrm{pb}^{-1}$.

\section{$2.2 n$ gluon exchange: Fits to $F_{2}$}

One of the primary goals of the HERA physics program is a precise measurement of the nucleon structure in a wide kinematic region. Both experiments have produced a wealth of new measurements ${ }^{3}$ of $F_{2}$ with relatively small systematic errors of $2-3 \%$. The kinematic range extends from momentum transfers, $Q^{2}$, of $0.1 \mathrm{GeV}^{2}$ to $5000 \mathrm{GeV}^{2}$ and Bjorken $x$ values in the range of $10^{-6}$ to 0.5 . The measurements are fit to parton densities parametrized at a given $Q_{0}^{2}$ and evolved to larger $Q^{2}$ using the GLAP evolution equation ${ }^{4}$ to NLO. The fits reproduce the data well over a large range of $Q^{2}$, including the measurements at values as low as $Q^{2}=1.5 \mathrm{GeV}^{2}$.

For kinematical reasons, the large $x$ values probed at HERA also correspond to large values of momentum transfers. As a consequence, $x g(x)$ is only weakly constraint at large $x$ by the present HERA data, due to lack of statistical precision. At low $x$ the extracted $x g(x)$ suffers from uncertainties related to the validity of the GLAP evolution, which ignores the $\ln 1 / x$ terms, the uncertainty in the subtraction of $F_{L}$, and the variations given by the choice of $Q^{2}$ threshold and functional parametrization of the parton densities. 
The new measurements of $F_{2}$ confirm the previous ${ }^{5}$ observation of a strong rise with decreasing $x$. The rise appears to be very steep at large $Q^{2}$ values and gradually flattens towards lower values of $Q^{2}$.

Under the assumption that the rise of $F_{2}$ with decreasing $x$ is driven by gluon splitting, $g \rightarrow q \bar{q}$, and that $x g(x)$ can be parametrized as $x^{-\lambda}$ at small $x$, H1 extracted the values ${ }^{6}$ of $\lambda$ as function of $Q^{2}$. The results of this phenomenological fit are shown by the points in Fig. 1 together with similar results obtained from fits to global data sets ${ }^{7}$. The data exhibit a clear increase in slope with $Q^{2}$, consistent with expectations from the GLAP evolution.

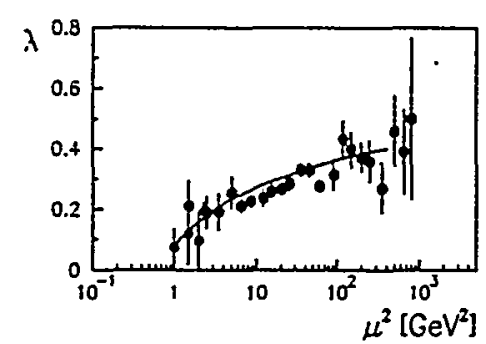

Figure 1. Slope of $F_{2}$ versus $Q^{2}$ as measured by $\mathrm{H} 1$. The solid line indieates the result obtained from a global fit to $F_{2}$ data?

\subsection{1 gluon exchange: Photon-gluon fusion process}

In the lower $Q^{2}$ range between 7 and $100 \mathrm{GeV}^{2}$ dijets are produced predominantly via the PGF process with a small background from QCD compton scattering (QCDC). The latter denotes the process where the scattered quark emits a hard gluon, resulting in two jets in the hadronic final state. In the LO approximation, the momentum fraction of the gluon emitted from the proton in PGF is reconstructed using the dijet mass, $m_{J J}$, and the kinematical variables of the event, $\xi=x_{B J}\left(1+m_{J J}^{2} / Q^{2}\right)$. At NLO the separation of PGF and QCDC is factorization scheme dependent and the momentum fraction of the gluon emitted from the proton is not identical to $\xi$. Therefore, $x g(x)$ can only be determined indirectly from the dijet rate, by adjusting, for instance, the input gluon density of the calculation until the measured and calculated dijet cross sections agree.

Fig. 2 shows a comparison of the measured and predicted differential cross section $d \sigma / d \xi$. The predictions are based on different input gluon densities with different slopes $\lambda$ and with the overall normalizations adjusted such, that at $Q^{2}=4 \mathrm{GeV}^{2} 42.7 \%$ of the proton momentum is carried by gluons. The data show a significant excess of about $34 \%$ compared to the predictions based on the standard MRSA ${ }^{8}$ parton densities with $\lambda=0.3$. This discrepancy is currently not understood. Uncertainties in the acceptance calculation, fragmentation models, and QCD scales typically result in $\pm 10 \%$ variations. Under the assumption that the shape is independent of the normalization, a value of $\lambda=0.38 \pm 0.04 \pm 0.18$ at $Q^{2}=4 \mathrm{GeV}^{2}$ can be extracted from fits to 
the shape of the $\xi$ dependence.

Figure 2. Differential dijet cross section versus $\xi$ as measured by ZEUS (preliminary). The histograms show the NLO prediction for input gluon densities with difierent slopes, $\lambda: 0.0$ (dashed), 0.3 (solid), 0.6 (dotted).

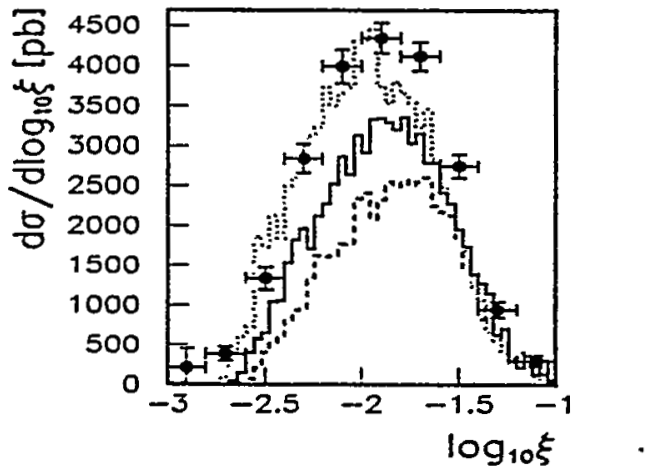

Both H1 and ZEUS have observed clean signals for open charm production in DIS, with about $100 D^{*}$ and 80 inclusive $D^{0}$ events ${ }^{9}$. The H1 collaboration studied the momentum fraction of the D's, $x_{D}$, to distinguish between intrinsic charm, as part of the proton, and extrinsic charm, produced via PGF. Due to the relatively low center-of-mass energy at HERA, the contribution to charm production from the fragmentation chain is negligible. The $x_{D}$ distribution is found to be soft and in agreement with expectations from the PGF production mechanism. MC studies using the charm quark densities of the proton obtained through massless evolution of the non-singlet parton densities predict a significantly harder distribution.

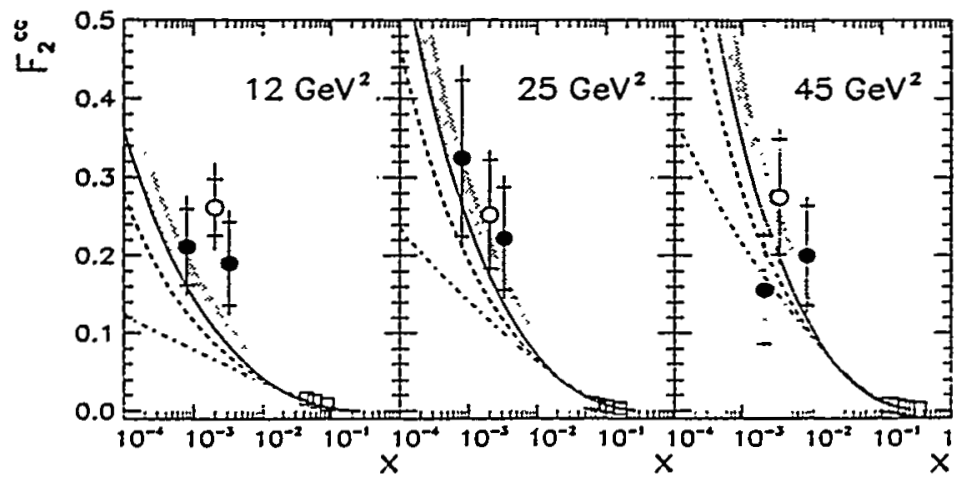

Figure 3. Fe versus $x$ for different $Q^{2}$ bins as measured by H1 using $D^{*}$ (closed dots) and $D^{3}$ (open dots), and EMC (open squares). The lines indicate the predictions based on the GRV HO (solid), MRS H (dashed), MRS DO (dot-dashed) parton densities. The band shows the predictions using the $\mathrm{HI}$ parton densities obtained from QCD fits to the measured $F_{2}$ values. 
This comparison is used to set a limit of $5 \%$ at $95 \%$ C.L. for intrinsic charm as the underlying production mechanism. Therefore, open charm production can be viewed as an almost background free sample of PGF events. Fig. 3 shows the measured $F_{2}^{c c}$ structure function in three different $Q^{2}$ bins. The measurement still lacks statistical precision, but the comparison with predictions based on different sets of parton densities shows a strong dependence on $x g(x)$.

To avoid confusion with the structure of real photons, both the dijet and the open charm techniques to measure $x g(x)$ are applied in the DIS regime. The method tagging PGF events using inelastic $\psi$ is, however, applied to the photoproduction sample, since the statistics in the DIS sample are currently too low. Cuts on the inelasticity of the $\psi$, defined as $z=E_{\psi} / E_{\gamma}$, where $E_{\psi}$ $\left(E_{\gamma}\right)$ is the energy of the $\psi$ (photon) in the photon-proton rest frame, are used to reject both elastic $\psi$ production and resolved photon events.

Fig. 4 shows the differential cross section for inelastic $\psi$ photoproduction versus $W$, the invariant mass of the photon-proton system. The measured cross sections are compared to predictions based on different input parton densities. To minimize the uncertainties due to higher order corrections beyond $\mathrm{NLO}$, an additional cut requiring $p_{\perp}^{\psi}>1 \mathrm{GeV} / \mathrm{c}$ has been imposed. Unfortunately, this additional cut also significantly reduces the sensitivity to $x g(x)^{10}$.

Figure 4. Differential cross section versus $W$ for inelastic $\psi$ photoproduction. The lines indicate predictions based on difierent parametrizetions of the parton densities of the proton.

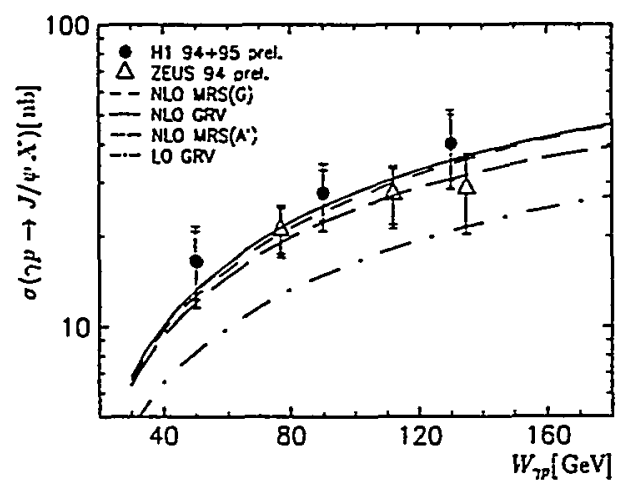

Assuming that the dependence of the cross section can be parametrized as $W^{a}$ and that $x g(x) \propto x^{-\lambda}$, then $\lambda=a / 2$. A fit to the data yields values of $\lambda=0.59 \pm 0.40(0.30 \pm 0.24)$ at a scale $\mu^{2}=m_{\psi}^{2} / 4$ for $\mathrm{H} 1$ (ZEUS).

\subsection{2 gluon exchange: Diffraction}

Exclusive vector meson production studies include measurements of $\rho, \omega, \phi$, and $\psi$ mesons. The exclusivity is imposed either by a cut on the calorimeter 
energy not associated with the meson and the scattered positron (ZEUS) or by a cut on the number of tracks (H1). Fig. 5 shows the $W$ dependence of the cross section for exclusive photoproduction of $\psi$ mesons.

Figure 5. Differential cross section versus $W$ for elastic $\psi$ photoproduction. The lines indicate predictions based on different parametrizations of the parton densities of the proton. The solid line represents a fit to the HERA data assuming a $W^{a}$ dependence.

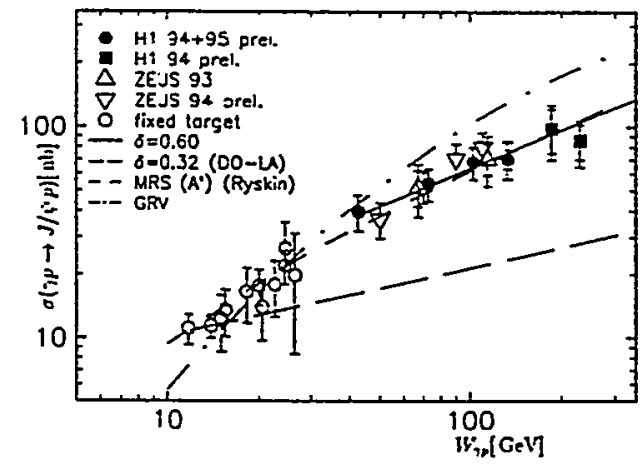

Under the assumption that the underlying production mechanism is dominated by the exchange of two gluons ${ }^{11}$, the cross section is expected to be proportional to $[x g(x)]^{2}$. This prediction neglects comparatively small corrections due to the transverse momentum $k_{t}$ of the gluons emitted from the proton. Thus, exclusive vector meson production offers a sensitive probe of $x g(x)$ at $x=\left(Q^{2}+m_{V M}^{2}\right) / W^{2}$ and scales, $\mu^{2}$, given by $m_{V M}^{2} / 4$ in photoproduction and $Q^{2}$ in DIS. Again, the slope of the $W$ dependence is, apart from the small corrections mentioned above, directly related to the slope $\lambda$ of $x g(x)$ : $\lambda=a / 4$.

Inclusive diffractive scattering at HERA is defined using three different definitions or requirements: i) the requirement of a rapidity gap in the hadronic system, ii) the requirement of a leading proton with at least $97 \%$ of the initial beam energy, and iii) the excess of events at low $\ln M_{X}^{2}$ over an exponentially falling background, where $M_{X}$ is the mass of the hadronic system as measured by the central detector. The three approaches give results which are not entirely consistent; for instance, the rapidity gap method ${ }^{12}$ finds a $W$ dependence which is constant with $Q^{2}$, whereas the low $\ln M_{X}^{2}$ method ${ }^{13}$ finds a slope that increases with increasing $Q^{2}$, see fig. 6 .

Assuming that diffraction is dominated by the exchange of two gluons, the $W$ dependence can be interpreted as a measurement of the slope of $x g(x)$, where $\lambda=\alpha_{P}-1$. 

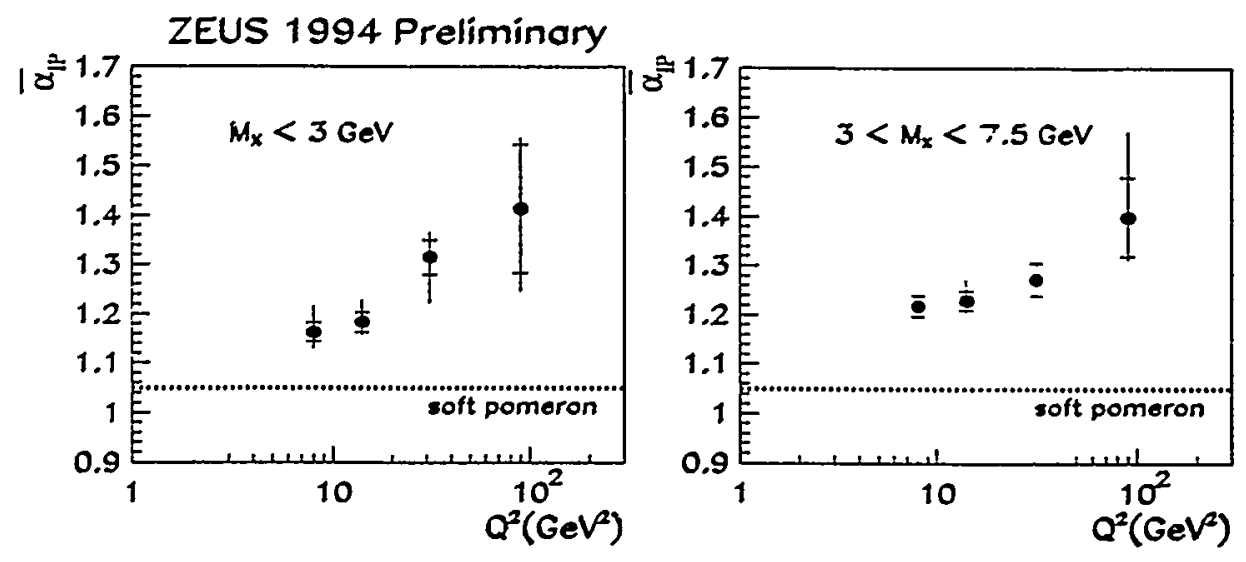

Figure 6. W dependence of the diffractive cross section versus $Q^{2}$ in two different bins of $M_{X}$ as measured by ZEUS (preliminary). The dependence is parametrized as $W^{4 a_{P}-4}$.

\section{Conclusions}

In summary, fig. 7 shows the approximate $x-Q^{2}$ ranges covered by the various methods. Clearly, the fits to $F_{2}$ cover the largest range, but the other methods provide a significant overlap and are essential to test the underlying physics model.

Figure 7. Comparison of the $x-$ $Q^{2}$ ranges covered by the different methods of extracting the gluon density.

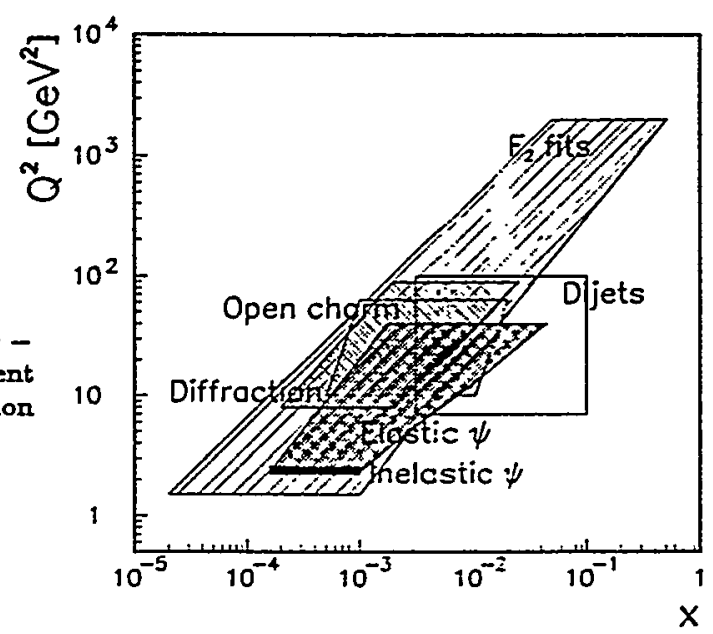

Fig. 8 compares the $\lambda$ values obtained from the different measurements: $F_{2}$ fits, dijet production, inelastic $\psi$ production, exclusive $\varphi$ production, and inclusive diffractive scattering. The overall agreement is quite satisfactory. 
Due to the lack of statistics, the slopes obtained from measurements of vector mesons other than the $\psi$, such as $\rho, \omega$, and $\phi$ have not been included. With higher precision, this type of comparison will provide a powerful tool to understand the various underlying production mechanisms and to determine the gluon density of the proton.

Figure 8. Comparison of the slope of $x g(x)$ as determined from fits to $F_{2}$ (open circles), dijet and $\psi$ production (filled triangles), and inclusive diffractive scattering (filled circles). The solid line indicates the result obtained from a global fit to $F_{2}$ data?

\section{Acknowledgements}

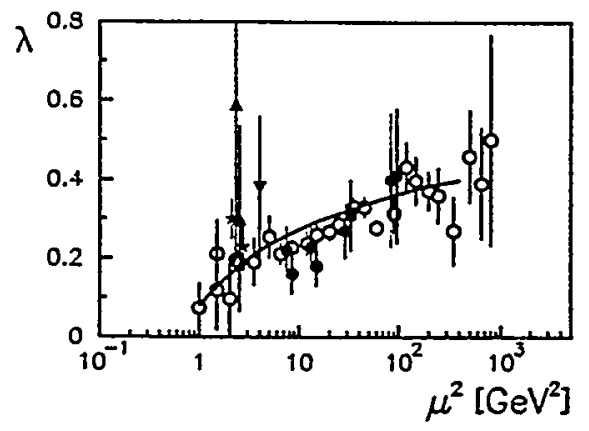

Many thanks to M.Derrick, D.Krakauer, and A.White for a careful scrutiny of the manuscript.

\section{References}

1. M. Klein and R.Yoshida, talks given at the International Workshop on Deep Inelastic Scattering and Related Phenomena, Rome, Italy (1996); H. Abramowicz, talk given at 28th International Conference on HighEnergy Physics (ICHEP 96), Warsaw, Poland (1996).

2. See, e.g., "Proceedings of the 3rd Workshop on Small-x and Diffractive Physics", Argonne National Laboratory, Argonne, IL (1996).

3. S.Aid et al, Nucl. Phys. B 470, 3 (1996); "Measurement of the $F_{2}$ Structure Function in Deep Inelastic $e^{+} p$ Scattering Using 1994 Data from the ZEUS Detector at HERA", M.Derrick et al, DESY-96-076, hepex/9607002.

4. V.N.Gribov and L.N.Lipatov, So. J. Nucl. Phys. 15, 438,675 (1972), L.N.Lipatov, Sov. J. Nucl. Phys. 20, 181 (1974), G.Altarelli and G.Parisi, Nucl. Phys. B 126, 297 (1977).

5. M.Derrick et al, Z. Phys. C 65, 379 (1995); T.Ahmed et al, Nucl. Phys. B 439, 471 (1995).

6. "A Measurement of the Proton Structure Function $F_{2}\left(x, Q^{2}\right)$ at low $x$ and low $Q^{2}$ at HERA", H1 collaboration, paper submitted to the 28th International conference on High Energy Physics (ICHEP 96), Warsaw, Poland (1996). 
7. "Determination of the gluon density", A.D.Martin, talk given at the International Workshop on Deep Inelastic Scattering and Related Phenomena, Rome, Italy (1996).

8. A.D.Martin, W.J.Stirling, and R.G.Roberts, Phys. Rev. D 50, 6734 (1994).

9. "Inclusive $D^{0}$ and $D^{* \pm}$ Production in Deep Inelastic ep Scattering at HERA", H1 collaboration, C. Adloff et al, DESY-96-138, hepex/9607012; " $D$ "士 Production in Deep Inelastic Scattering at HERA", ZEUS collaboration, paper submitted to the 28th International conference on High Energy Physics (ICHEP 96), Warsaw, Poland (1996).

10. M.Krämer, Nucl. Phys. B 459, 3 (1995).

11. M.G.Ryskin, Z. Phys. C 57, 89 (1993), M.G.Ryskin et al, DTP-95-96, hep-ph/9511228.

12. "Diffraction in DIS", P.Biddulph (H1), talk presented at the 3rd Workshop on Small-x and Diffractive Physics, Argonne National Laboratory, Argonne, IL (1996).

13. "Diffraction in DIS", H.Kowalski (ZEUS), talk presented at the 3rd Workshop on Small-x and Diffractive Physics, Argonne National Laboratory, Argonne, IL (1996). 